\title{
Ökonomisierung gelungen, Pflegekräfte wohlauf?
}

Gesundheitsreform und kein Ende. Täglich erreichen uns neue Pläne und Reformvorschläge zur „Sanierung“ des deutschen Gesundheitssystems. Auch vor den Krankenhäusern hat diese Entwicklung nicht halt gemacht - zentrale Stichpunkte sind die Privatisierung von Krankenhäusern und die Umstellung der Krankenhausfinanzierung auf ein Fallpauschalensystem. Davon betroffen sind nicht nur die Patienten, sondern auch die Beschäftigten, die ihren Arbeitsplatz im Krankenhaus haben. Das Interesse dieses Artikels richtet sich auf die Pflegekräfte: Welche Folgen hat die Ökonomisierung des Gesundheitswesens für ihre Arbeitssituation?

\section{1 Reformen ohne Ende -
Wo stehen die Pflegekräfte?}

Aktuell durchläuft die stationäre Gesundheitsversorgung in Deutschland einen Strukturwandel hin zu mehr Wettbewerbsund Leistungsorientierung. Die Krankenhäuser sehen sich aufgrund veränderter ökonomischer und politischer Rahmenbedingungen mit einem zunehmenden Kosten- und Leistungsdruck konfrontiert. Dieser Wandel wird unter dem Schlagwort der Ökonomisierung des Krankenhaussektors breit und vor allem kontrovers diskutiert. Mit dem Begriff der „Ökonomisierung“ ist in diesem Kontext gemeint, dass das patientenbezogene Denken und Handeln in Krankenhäusern zunehmend durch eine betriebswirtschaftliche Handlungslogik beeinflusst wird, medizinisch-pflegerische Versorgungsziele werden durch wirtschaftliche Kalküle und Ziele überformt. Daher ist Ökonomisierung auch kein Synonym für Wirtschaftlichkeit, denn sie kann empirisch sowohl wirtschaftliche als auch unwirtschaftliche Folgen haben (Kühn 2003; Kühn/Klinke 2006).

Im Zuge der fortschreitenden Ökonomisierung des Krankenhaussektors unterliegen auch die Organisations- und Steuerungsformen von Kliniken einem grundlegenden Wandel: Moderne Managementkonzepte werden etabliert, Controllingund Benchmarksysteme gewinnen an Bedeutung, Kennziffern und ergebnisorientierte Steuerungsformen halten Einzug in den Klinikalltag - an und für sich nichts Neues? In der Tat, diese neuen (marktorientierten) Steuerungsformen sind uns aus anderen Bereichen längst bekannt (Wagner 2005; Sauer 2005; Dörre/Röttger 2003).
Dennoch konnte dem Gesundheitswesen im Vergleich zu herkömmlichen Unternehmen bislang eine gewisse „Eigenlogik“ unterstellt werden. Und genau diese markiert systemimmanente Grenzen der Ökonomisierung des Krankenhauswesens, wie dieser Beitrag aufzeigen wird. ${ }^{1}$

Im Folgenden richtet sich der Fokus auf die Pflegekräfte in sogenannten Akutkrankenhäusern. Obwohl sie zahlenmäßig die größte Berufsgruppe im Krankenhaus ausmachen, nehmen sie - im Vergleich zu den Ärzten - in der öffentlichen Debatte zum Wandel des Gesundheitswesens bislang keine prominente Stelle ein. Dennoch stehen auch ihre Arbeitsanforderungen und Arbeitsaufgaben im Zuge des Strukturwandels unter Veränderungsdruck und damit steht zugleich der innerste Kern ihrer beruflichen Identität infrage. ${ }^{2}$

\section{Symptome der \\ Ökonomisierung}

Zwei wesentliche Veränderungstendenzen kennzeichnen den gegenwärtigen Umbruch der Krankenhauslandschaft und können zugleich als Symptome der seit mehreren Jahren andauernden Ökonomisierung des deutschen Gesundheitswesens betrachtet werden: erstens die zunehmende Privatisierung der Krankenhauslandschaft und zweitens die bislang weitestreichende Krankenhausreform des deutschen Gesundheitswesens - die schrittweise Umstellung der Krankenhausvergütung auf ein Fallpauschalensystem (DRG). Setzt sich im Zuge dieser Veränderungstendenzen eine „neue Herrschaft der Zahlen“ im Krankenhauswesen durch?

\subsection{PRIVATISIERUNG VON KRANKENHÄUSERN}

Der Krankenhausmarkt in Deutschland befindet sich in Bewegung: Privatwirtschaftlich tätige, teilweise börsennotierte Klinikketten kaufen den Kommunen ihre wirtschaftlich defizitären Krankenhäuser nach und nach ab. Das schlägt sich auch in den verfügbaren Statistiken nieder: Zwischen 2004 und 2005 wuchs der Anteil privater Krankenhäuser um rund $10 \%$, während der Anteil der öffentlichen Krankenhäuser

\begin{tabular}{l}
\hline Der Beitrag beruht auf den empirischen Ergebnis- \\
sen des laufenden Forschungsprojekts "Arbeit \\
unter Marktdruck - Neue Steuerungsformen und \\
Leistungspolitik", das von der Hans-Böckler-Stif- \\
tung gefördert und gegenwärtig am ISF München \\
durchgeführt wird. Zielsetzung dieses empirischen \\
Forschungsprojekts ist die Analyse der Leistungs- \\
steuerung in verschiedenen Dienstleistungsberei- \\
chen. \\
\hline Die empirische Basis der folgenden Ausführungen \\
bilden Erhebungen in zwei verschiedenen Kliniken \\
eines großen kommunalen Klinikums der maxima- \\
len Leistungsstufe. Es gilt als innovativ und wirt- \\
schaftlich erfolgreich. Die Mitarbeiter genießen \\
auf der Basis eines Beschäftigungspakts Schutz vor \\
betriebsbedingter Kündigung. Auch Gehaltsein- \\
bußen und tarifliche Notlagenverträge konnten \\
bislang ausgeschlossen werden. \\
Ingesamt wurden Interviews mit 30 Personen ge- \\
führt: Intensivinterviews mit Gesundheits- und \\
Krankenpflegerinnen und -pflegern ergänzt durch \\
Expertengespräche mit Vertretern des Manage- \\
ments und des Personalrats sowie der Gewerk- \\
schaften.
\end{tabular}

Kira Marrs, Dr., ist Wissenschaftlerin am Institut für Sozialforschung (ISF) München. Arbeitsschwerpunkte: Veränderung von Leistungspolitik, Entwicklung von Dienstleistungsarbeit.

e-mail: kira.marrs@isf-muenchen.de 
um knapp $4 \%$ abnahm. ${ }^{3}$ Entgegen dem Branchentrend konnten die großen privaten Krankenhausketten, wie Asklepios-Kliniken, Helios-Kliniken oder Rhön-Klinikum, Zuwächse in Bezug auf Unternehmensgröße, Umsatz und sogar Gewinn verzeichnen. So steigerte die Rhön-Klinikum AG im Jahr 2005 ihren Umsatz um rund $35 \%$ - wohl nicht zuletzt durch die Übernahme von insgesamt elf Kliniken innerhalb dieses Zeitraums. Für die kommunalen Krankenhäuser bedeuten diese Marktverschiebungen sowie die Etablierung privater Klinikketten eine neue Wettbewerbssituation. Ein befragter Experte eines kommunalen Krankenhauses charakterisiert sie mit den Worten, es gehe „ums Überleben“.

Neben Aufkäufen und Fusionen wird aktuell auch ein "Kliniksterben" 4 diagnostiziert - eine Entwicklung, die sich im Laufe der nächsten Jahre weiter zuspitzen dürfte. Nach Schätzung der Deutschen Krankenhausgesellschaft aus dem Jahr 2004 werden in den nächsten zehn Jahren rund $15 \%$ aller Krankenhäuser in Deutschland, das sind immerhin 330 Krankenhäuser, für immer ihre Pforten schließen müssen. Radikalere Prognosen nehmen an, dass bis zum Jahr 2015 jede vierte Klinik ihren Betrieb einstellen muss.

\subsection{EINFÜHRUNG DIAGNOSE- BEZOGENER FALLPAUSCHALEN}

Mit der Einführung einer neuen Form der Leistungsabrechnung und -finanzierung der Krankenhäuser findet die bislang umfassendste Krankenhausreform im deutschen Gesundheitswesen statt. Im Jahr 2003 wurde die Krankenhausvergütung auf ein Fallpauschalensystem umgestellt, das sich an dem international bereits etablierten DRG-System (Diagnosis Related Groups) orientiert. Für den Finanzierungsmodus der Krankenhäuser zählt jetzt nicht mehr die Liegezeit, sondern die Fallpauschale. ${ }^{5}$

Durch die DRGs wird für eine bestimmte Erkrankung eine Pauschalvergütung, also ein Festpreis, bezahlt - unabhängig von den individuellen Aufwendungen und unabhängig von dem Krankenhaus, in dem sie erbracht werden. D.h. der "Preis“ für eine Hüftoperation ist ebenso festgelegt wie der für eine bestimmte Herzoperation; die tatsächliche Liegezeit eines Patienten spielt zukünftig für die Leistungsabrechnung der Krankenhäuser eine nach- geordnete Rolle. Dementsprechend ist das erklärte Ziel dieser Reform die deutliche Reduktion der Verweildauer von Patienten.

Die DRGs sind allerdings weit mehr als nur ein neues Entgeltsystem der Krankenhausfinanzierung. Sie sind zugleich ein umfassendes Klassifikationssystem zur Definition und Bewertung von Krankenhausleistungen. Auf der Basis einer „informatorischen Durchdringung" (Boes 2005) des Versorgungsgeschehens werden medizinische und ökonomische Daten erzeugt, die eine neue Transparenz und Vergleichbarkeit von Krankenhausleistungen schaffen (Kühn/Klinke 2006). Diese werden dadurch einem umfassenden und detaillierten Controlling zugänglich und können somit hinsichtlich ihrer Effizienz und ihrer Kosten auf unterschiedlichen Ebenen analysiert und wirtschaftlich optimiert werden: Einzelne Behandlungsfälle, Stationen und Kliniken können Kostenvergleichen und Benchmarking-Prozeduren unterworfen werden (Vogd 2006).

Die DRGs bilden somit die Grundlage für die verstärkte Ökonomisierung von Krankenhäusern und setzen zugleich den Anreiz für eine stärker betriebswirtschaftliche Ausrichtung (Kühn/Simon 2001; Kühn 2003; Braun/Müller 2006). Dies betrifft nicht nur die Überprüfung bisheriger Strukturen und Betriebsabläufe mit dem Ziel, die Kostenstrukturen zu rationalisieren und zu verschlanken, sondern es werden zugleich neue prozess- und ergebnisorientierte Formen der Unternehmenssteuerung über Zielvorgaben und Kennziffern etabliert (Kühn/Klinke 2006).

\subsection{EINE "NEUE HERRSCHAFT DER ZAHLEN" IN KRANKENHÄUSERN?}

Die fortschreitende Ökonomisierung des Gesundheitswesens stellt neue Herausforderungen an die Steuerung und Führung von Krankenhäusern. Vormals praktikable Ansätze und Konzepte versagen unter dem zunehmenden Kosten- und Leistungsdruck. Den meisten Krankenhäusern fehlt es auch an ausreichenden Erfahrungen im Umgang mit dynamischen Wettbewerbsbedingungen. Demgegenüber hat das untersuchte kommunale Klinikum frühzeitig versucht, den Strukturbrüchen im Gesundheitswesen mit neuen Managementkonzepten zu begegnen. Ende der 1990er Jahre wurde das städtische Krankenhaus in ein selbstständiges gemeinnütziges Kommunalunternehmen unter städtischer Trä- gerschaft umgewandelt. Dem Wandel der Rechtsform von einem Regiebetrieb in eine $\mathrm{GmbH}$ folgte eine weitreichende Strukturreform des Klinikums. Die Klinikumsleitung wurde reorganisiert, die einzelnen Klinken wurden in dezentrale Organisationseinheiten mit großen Handlungsspielräumen und eigener Budgetverantwortung umstrukturiert, welche seither über interne Budgetierung und operative Kennziffern von der Klinikumsleitung in Kooperation mit dem Controlling gesteuert und kontrolliert werden. Seit der Umstellung der Krankenhausfinanzierung auf Fallpauschalen sind die DRG-Leistungskennziffern, wie durchschnittliche Verweildauer oder DRG-Fallzahl, zu zentralen Kennziffern der Unternehmenssteuerung avanciert.

Früher hatte der kommunale Träger aufkommende Defizite relativ bereitwillig übernommen, doch nun stehen die Kliniken unter erheblichem Druck, ihre wirtschaftlichen Zielvorgaben $\mathrm{zu}$ erfüllen. Schließlich kann vom Erreichen dieser Ziele der Erhalt der Klinik abhängen. So droht beispielsweise bei wirtschaftlichem Misserfolg eine Verkleinerung der Klinik durch Bettenabbau, Stationszusammenlegungen und Stationsschließungen. Aber nicht nur wirtschaftlicher Misserfolg, sondern auch Erfolg kann negative Konsequenzen nach sich ziehen. Aufgrund ihres sehr erfolgreichen Abschneidens in den letzten Jahren wurden bei einer der untersuchten Kliniken die Ziele sukzessive erhöht, und bislang scheint bei dieser Zieldynamisierung noch nicht das Ende der Fahnenstange erreicht zu sein. In einer anderen untersuchten Klinik wird seit mehreren Jahren das Managementsystem der Balanced Scorecard im

3 Nach Angabe der Deutschen Krankenhausgesellschaft befanden sich im Jahr 2005 bereits mehr als $26 \%$ der Krankenhäuser in privater Trägerschaft; der Anteil öffentlicher Krankenhausträger betrug knapp $35 \%$, der von freigemeinnützigen Trägern $38 \%$ (http://www.dkgev.de/pdf/1570.pdf).

4 Aktuelle Informationen finden sich zu diesem Thema im Internet unter der Adresse: www. kliniksterben.de. Weiteres unter http://www.welt.de/ printwams/article108662/Gesundheitsreform_fuehrt_ zu_Krankenhaussterben.html, bzw. http://www. arthurandersen.de.

5 Die Umstellung auf Fallpauschalen erfolgt schrittweise. Gegenwärtig befinden sich die Krankenhäuser in einer bis 2009 laufenden Konvergenzphase. So lange erhalten die Kliniken ein Budget, aus dem sich die klinikindividuellen Fallpauschalen errechnen, danach sollen diese landesweit einheitlich sein (Laschet 2007). 
Kontext eines Qualitätsmanagementsystems eingesetzt. Balanced Scorecard, was in etwa „ausgewogenes Berichtssystem“ bedeutet, beabsichtigt eine ganzheitliche und strategische Steuerung des Unternehmens, orientiert an einem mehrdimensionalen Zielsystem entlang der vier Dimensionen Finanzen, Kunden, Prozesse und Mitarbeiter (Braun von Reinersdorff 2002; Pfaff et al. 2004).

Diese Zielvorgaben und Kennziffern der Kliniken spiegeln sich bislang (noch) nicht auf der Mitarbeiterebene in leistungspolitischen Instrumenten wider, wie beispielsweise Zielvereinbarungen oder variables Leistungsentgelt. Zielvereinbarungen werden nur hinsichtlich qualitativer Ziele, wie z. B. die Einführung einer Wunddokumentation oder die Überarbeitung des Nachtdienstkonzepts mit den Pflegekräften, abgeschlossen. Im Verlauf des Jahrs 2007 wird sich aber mit der Umsetzung des neuen Tarifvertrags des öffentlichen Dienstes (TVöD) eine Leistungsorientierung in den Entgeltstrukturen niederschlagen (Tondorf 2007). Im Gegensatz zum vormals geltenden Bundesangestelltentarifvertrag (BAT) sieht dieser erstmalig leistungsvariable Entgeltbestandteile für kommunale Krankenhäuser vor. Unklar ist bislang, wie krankenhausspezifische Kriterien der Leistungsmessung und Leistungsbeurteilung überhaupt gewonnen werden können (Hartwig 2002). Mit dieser Thematik setzen sich auch die Pflegekräfte kritisch auseinander: Wie kann Leistung im Pflegebereich definiert, bewertet und vergütet werden?

\section{Pflegearbeit unter Verän- derungsdruck - Perspek- tiven der Pflegekräfte}

Jeder, der einen längeren Aufenthalt im Krankenhaus als Patient verbracht hat, weiß aus eigener Erfahrung, dass die Arbeit einer Pflegekraft kaum vergleichbar mit Tätigkeiten in anderen Wirtschaftssektoren ist. Bei dieser personenbezogenen Dienstleistungstätigkeit wird eine eigene Qualität von Arbeit gefordert: Für die Krankenpflege ist der äußerst enge Kontakt zu den Nutzern der Arbeit konstitutiv (Rieder 1999), und die Erbringung der Dienstleistung erfordert die persönliche Begegnung, die Kommunikation und ein gewisses Maß an
Kooperation zwischen Patient und Pflegenden. Demnach kann der Kernbereich der Pflegetätigkeit auch als „praktizierte Zwischenmenschlichkeit" bestimmt werden (Badura 1990). Die gesellschaftlichen Leitbilder zur Pflege spiegeln sich auch in den beruflichen Orientierungen vieler Pflegekräfte wider, zumindest beim Berufseinstieg. Internationale Studien diagnostizieren seit Langem relativ konsistente Motive der Berufswahl für Pflegekräfte: So sind der Wunsch zu helfen und der Wunsch, mit Menschen zu arbeiten, zentrale Motive für die Berufswahl (Dunkel 2005).

Die berufliche Praxis kann diesen hohen Ansprüchen häufig nicht gerecht werden: Viele Pflegekräfte nennen als Grund für das Nachdenken über einen Berufswechsel, dass ihre Arbeitsbedingungen ihnen keine gute Pflege ermöglichen; sie klagen über fehlende Zeit für ihre Patienten (Braun et al. 2004). Insbesondere die hohen Belastungen der beruflichen Pflegearbeit werden als zentrale Ursache für die hohe Fluktuation innerhalb des Berufsstandes und die hohe Krankheitsrate im Pflegeberuf angesehen. Im Vergleich zur Allgemeinbevölkerung weisen Pflegekräfte nämlich ein deutlich erhöhtes Gesundheitsrisiko auf (Zimber 2003). Es entsteht durch körperliche Belastungen (z.B. Heben, Drehen und Lagern von Patienten), durch Infektionserreger und gefährliche Stoffe sowie durch psychomentale Belastungen: Zeitdruck, aber auch Miterleben von Krankheit, Leiden und Tod. Beanspruchungen resultieren zudem aus den Arbeitszeitstrukturen, die sich am 24-Stunden-Betrieb eines Krankenhauses orientieren - und das bedeutet für die Mehrzahl der Pflegekräfte Wechselschicht mit Nachtdienst (Michaelis 2005).

Pflegearbeit ist unter den heutigen Bedingungen eine hochgradig verdichtete Tätigkeit: Hektik, Stress und starker Zeitdruck kennzeichnen die Arbeitssituation vieler Pflegekräfte. Vor diesem Hintergrund stellt sich die Frage, welche Folgen ökonomische und sachliche Zwänge des Gesundheitswesens für die Arbeitssituation und die Motivation der Pflegekräfte haben.

\subsection{VERÄNDERUNG DER ARBEITS- SITUATION}

Die befragten Pflegekräfte erlebten in den vergangenen Jahren eine deutliche Veränderung ihrer Arbeitssituation - diese Einschätzung wird im Übrigen auch vom
Management bestätigt. Nicht nur die Pflegekräfte berichten von einer zunehmenden Arbeitsverdichtung und einem erhöhten Zeit- und Leistungsdruck, auch ihre Vorgesetzten geben an, dass sich die Arbeits- und Leistungssituation der Pflegekräfte negativ verändert hat und dass in manchen Bereichen über Leistungsreduzierung nachgedacht werden muss. Ein Indikator für die Arbeits- und Leistungssituation vieler Pflegekräfte ist, dass sie nach Aussage von Vertretern des Personalrats und des Managements ihren Beruf unter den heutigen Bedingungen voraussichtlich nicht bis zum Renteneintrittsalter werden ausüben können.

Aus der Sicht der Pflegekräfte können zwei zentrale Veränderungsmomente ihrer Arbeit im Krankenhausalltag benannt werden, die beide in engem Zusammenhang mit der DRG-Einführung stehen: Sie beobachten erstens eine zunehmende Bürokratisierung ihrer Arbeit. Der Anteil der Dokumentations- und Verwaltungsaufgaben an ihrer Gesamtarbeitszeit hat sich drastisch ausgeweitet. Die ausführliche Dokumentation erfolgt nicht allein zur rechtlichen Absicherung gegenüber Ansprüchen von Patienten und Angehörigen, sondern auch zur Sicherung und Optimierung der Erlöse im DRG-System. Fast jeder Handgriff muss mittlerweile dokumentiert werden, egal ob Haare gekämmt werden, eine Seitenlagerung vorgenommen oder Inkontinenz versorgt wird. D.h. es liegt ein besonderes Augenmerk auf der umfassenden und detaillierten Dokumentation aller erbrachten pflegerischen Leistungen. Gerade im Kontext der DRGs geht es hier wirklich um „Geld“, wie es eine befragte Pflegekraft formulierte. Diese Bürokratisierung der Arbeit wird überwiegend negativ von den Pflegekräften bewertet und als lästig empfunden (Braun et al. 2004), da es Zeit ist, die ihnen für die direkte Arbeit am Patienten fehlt.

Zweitens nannten die befragten Pflegekräfte Arbeitsverdichtung und Erhöhung des Arbeitstempos als wesentliche Veränderungen ihrer Arbeitssituation. Auch diese Entwicklungen stehen in engem Zusammenhang mit der fortschreitenden Ökonomisierung. Das DRG-System setzt ökonomische Anreize für eine Verkürzung der Liegezeiten von Patienten und eine Erhöhung der Fallzahlen. Beide Kennzahlen, Verweildauer und Fallzahlen, sind zugleich zentrale Stellschrauben für die Arbeitsund Leistungssituation der Pflegekräfte. Es 
ist unmittelbar einleuchtend, dass es zur Beschleunigung und Verdichtung der Arbeit kommt, wenn mehr Patienten in kürzerer Zeit versorgt und behandelt werden. So ist der pflegerische Aufwand pro Patient über die Dauer seines Krankenhausaufenthalts nicht konstant. Der durchschnittliche Aufwand ist in den ersten drei Tagen besonders hoch und geht dann in der Regel sukzessive bis zu seiner Entlassung zurück. Von zentraler Bedeutung ist hierfür natürlich die eintretende Genesung des Patienten. Neben dem Gesundheitszustand spielt aber auch der Verwaltungsaufwand, der für jeden Patienten, unabhängig von der Dauer seines Aufenthaltes, erledigt werden muss, eine wichtige Rolle. Bei jedem Neuzugang muss beispielsweise der Gesundheitszustand akribisch festgehalten werden, um eine adäquate Versorgung gewährleisten zu können, aber auch um im Nachhinein beweisen zu können, dass z. B. der Dekubitus nicht während des Krankenhausaufenthalts entstanden ist, sondern bereits vor der Einweisung bestand und dementsprechend z. B. dem Altenheim anzulasten ist. Ferner müssen die erforderlichen Untersuchungen dergestalt organisiert werden, dass eine schnellstmögliche Entlassung erfolgen kann. Für die Pflegekräfte bedeutet das einen erhöhten Koordinationsaufwand und mehr Zeitdruck. Dabei können ganz pragmatische Probleme auftreten: Wann soll etwa die tägliche Grundpflege der einzelnen Patienten erfolgen, wenn diese permanent bei den unterschiedlichsten Untersuchungen sind?

Zusammenfassend kann festgehalten werden, dass die Arbeitssituation der Pflegekräfte durch die ökonomischen und politischen Veränderungen beeinflusst wird und dass der Strukturwandel (un)mittelbare Auswirkungen auf den pflegerischen Dienst hat (Braun et al. 2004; Bartholomeyczik 2007).

\subsection{INTEGRATION ÖKONOMISCHER PRINZIPIEN IN DIE PFLEGERISCHE PERSPEKTIVE?}

In der aktuellen Debatte über den Strukturwandel des Gesundheitswesens nimmt die Forderung nach einer stärkeren Berücksichtigung von ökonomischen Aspekten bei der Leistungserbringung viel Raum ein. Davon betroffen sind auch die Pflegekräfte - sie sollen ein stärkeres Bewusstsein für Kosten und Nutzen einzelner Maßnahmen und Entscheidungen entwickeln, sie sollen betriebswirtschaftliche Kompetenz entfalten. In dem untersuchten Klinikum wird der Versuch unternommen, ein neues Leistungsspektrum in der Pflege zu etablieren. Die Zielsetzung lautet, pflegerische Primärverantwortung mit betriebswirtschaftlicher Kompetenz zu verbinden. Demnach soll die Tätigkeit von Pflegefachkräften weit mehr als „nur“ die Grund- und Behandlungspflege umfassen. Das Leistungsspektrum sieht zusätzlich vor: betriebswirtschaftliche Verantwortung, Managementfunktion, Spezialisierung bis hin zur Pflegewissenschaft, Datenverarbeitung, dezentrales Controlling und Fallmanagement. Nach den Vorstellungen und Wünschen der Klinikleitung sollen die Pflegekräfte zu Promotoren einer neuen Wettbewerbs- und Leistungsorientierung im Krankenhaus avancieren und wirtschaftliches Handeln und Denken in ihrem beruflichen Selbstverständnis verankern. Hierzu die Aussage eines befragten Experten: „Die Primary Nurse ${ }^{6}$ muss pflegerische Vorgaben machen, Kontrolle, Überwachung, organisieren, managen. Und dazu gehört auch, nicht ein Röntgen anmelden, sondern Röntgen anmelden und sagen: Wann geht's? Und wenn der andere rumzickt, sofort sagen: Sie vergeuden meine Arbeitszeit, das Röntgen muss raus, wir haben mittlere Verweildauer der DRG. Sie muss zur Managerin werden, zur Organisatorin des Ganzen."

Bevor den Fragen nachgegangen wird, ob Pflegekräfte tatsächlich zu Managern arrivieren möchten und ob sie dies mit ihrem beruflichen Selbstverständnis vereinbaren können, ist zu analysieren, ob ihnen aktuell überhaupt die Möglichkeit gegeben wird, zu Akteuren der Leistungssteuerung $\mathrm{zu}$ avancieren. Viele der befragten Pflegefachkräfte, vor allem die Stationsleiter, kritisieren nämlich, dass ihnen keine ausreichenden Steuerungsmöglichkeiten zur Verfügung stünden. Obwohl im Zuge der Strukturreform die Budgetverantwortung auf die einzelnen Kliniken, die seither auch als dezentrale Organisationseinheiten bezeichnet werden, delegiert wurde, sind diese nach Aussage einiger Interviewpartner „zahnlose Tiger“, wenn es um die Steuerung geht. So bietet das Berichtswesen des Controllings aufgrund der hohen Anzahl von Fehlern häufig nicht die erforderliche Informationsgrundlage - es wird von manchen Stationsleitern gar als Placebo oder Feigenblatt des dezentralen Controllings bezeichnet. Darüber hinaus sind die Mög- lichkeiten zur Steuerung durch gesetzliche und ethische Bestimmungen und Rahmenbedingungen entscheidend beeinflusst. So stoßen betriebswirtschaftliche Ziele und Kalküle im patientenbezogenen Handeln gerade bei schwerstkranken Patienten schnell an Grenzen. „Ich kann nicht das Beatmungsgerät ausschalten " argumentierte eine befragte Stationsleitung einer Intensivstation. Entgegen den Vorstellungen mancher Betriebswirtschaftler fehle ihnen speziell bei dieser Patientengruppe eine Handhabe, anfallende Kosten einzuschränken. Der Stationsleiter verdeutlicht damit sehr plastisch den Widerspruch zwischen ökonomischen Zielsetzungen und ethischmoralischen Pflichten - sein Fazit lautet „der Mensch ist dann einfach teuer“.

Das DRG-System setzt deutlich stärkere Anreize für die wirtschaftliche Behandlung der Patienten, als dies früher der Fall gewesen ist. Zugespitzt formuliert, steht nicht mehr allein der kranke Mensch und sein individuelles Schicksal im Mittelpunkt, sondern auch der Erlös, der aus der Behandlung resultiert. Die Frage, ob für Pflegekräfte die Erlösoptimierung und das Kundenparadigma im Klinikalltag orientierungs- und motivationswirksam sind, kann anhand unserer empirischen Befunde folgendermaßen beantwortet werden: Unterhalb der Stationsleitungsebene ist relativ wenig davon bei den Pflegekräften angekommen. Sie sehen es - zumindest bislang - nicht als ihre Aufgabe an, einen eigenständigen Beitrag zur Gewinnerzielung zu leisten. Eine befragte Pflegekraft einer Intensivstation äußerte ganz unverblümt ihre Haltung gegenüber erlösoptimierenden Handlungs- und Verfahrensweisen auf Seiten der Pflegekräfte - das sei nicht ihr Job, sie interessiere sich für ihre Patienten.

\footnotetext{
6 "Primary Nursing" bezeichnet eine aus den USA stammende Form der Pflegeorganisation. Eine Pflegekraft übernimmt die "Rund-um-die-UhrVerantwortung " für einen Patienten und begleitet diesen von der Aufnahme bis zur Entlassung (Tewes 2006). Im untersuchten Klinikum soll in Zukunft die "Primary Nurse" durch pflegerische As sistenzkräfte unterstützt werden, welche z. B. Servicetätigkeiten übernehmen sollen. Faktisch könnte dadurch die bislang bestehende nicht-arbeits teilige und ganzheitliche Pflegeorganisation, die sogenannte Bereichspflege (u.a. Büssing 1997), durch ein hierarchisches Pflegesystem abgelöst werden. Zugleich ändert sich auch die Qualifikationsstruktur - durch die Übertragung von Tätigkeiten höherer Entgeltgruppen auf niedrigere Entgeltgruppen kommt es zu Einsparungspotenzialen.
} 


\subsection{BERUFLICHES SELBSTVERSTÄND- NIS UND ÖKONOMISIERUNG - EIN WIDERSPRUCH?}

Für die Pflegekräfte stellt die zunehmende Dominanz der Ökonomie im Gesundheitswesen einen äußerst widersprüchlichen Prozess dar (Kühn/Simon 2001), der ihre Wahrnehmungen und Orientierungen, ihre Handlungsperspektiven und ihr berufliches Selbstverständnis berührt. Viele Pflegekräfte gehen mit hohen ethisch-moralischen Ansprüchen an ihre Berufstätigkeit heran. Besteht für die befragten Pflegekräfte ein Konflikt zwischen der zunehmenden Ökonomisierung des Krankenhauswesens und ihrem beruflichen Selbstverständnis? Für die Mehrheit der befragten Personen kann konstatiert werden, dass die Ökonomisierung der Pflege die berufliche Motivation, aus der heraus sie ihren Beruf praktizieren, beeinträchtigt. So beschrieb beispielsweise eine Pflegekraft einer Allgemeinstation sehr eindringlich, dass es für sie persönlich ungeheuer schwierig sei, ihren Anspruch, Menschen zu helfen und sie optimal zu versorgen, mit den Bedingungen des Stationsalltags in Einklang zu bringen. Das schnelle Durchschleusen von Patienten und das Arbeiten unter enormem Zeitdruck konterkariere ihre Vorstellungen einer „guten Pflege“ - das passe ihrer Ansicht nach nicht gut zusammen. D.h. gerade weil die hohe Arbeitsmotivation vieler Pflegekräfte durch ihr Interesse an der Qualität ihrer Arbeit begründet ist, geraten sie in einen systematischen Konflikt mit den aktuellen Ökonomisierungsbestrebungen im Krankenhauswesen.

Insbesondere das DRG-System bietet laut Aussage unserer Interviewpartner nicht nur Anlass zu kritischen Diskussionen und Befürchtungen, sondern auch zu Gewissenskonflikten. Die befragten Pflegekräfte äußerten die Vermutung, dass das neue Fallpauschalensystem vorzeitige Entlassungen von Patienten befördere. Das Stichwort in diesem Kontext ist „blutige Entlassung". Außerdem stelle sich der sogenannte „Drehtüreneffekt" mit zu schnellen Entlassungen und Wiedereinweisungen ein, wodurch nicht nur die davon betroffenen Patienten, sondern auch die Wirtschaftlichkeit des Systems belastet werde. Diese Ökonomisierungstendenz des Krankenhauswesens kann aber nicht nur für Patienten negative Folgen haben, sondern stellt auch das berufliche Selbstverständnis von Pflegenden infrage. So argumentierte ein befragter Stationsleiter, dass insbesondere der „Drehtüreneffekt" die Unsinnigkeit des Systems offenbare, das im Widerspruch zum beruflichen Selbstverständnis vieler Pflegekräfte stehe und Anlass zu Gewissenskonflikten im Stationsalltag gebe. Schließlich hätten die allermeisten Pflegekräfte ihren Beruf aus „Liebe zum Menschen" ergriffen. Ohne diesen spezifischen Sinngehalt ihres Berufs wäre es hingegen vernünftiger - so der befragte Stationsleiter - einer Tätigkeit mit besseren Verdienstmöglichkeiten und geringeren Belastungen nachzugehen.

Es bleibt festzuhalten, dass die wachsende Erlös- und Gewinnoptimierung für die Pflegenden eben nicht orientierungsund motivationswirksam ist. Der gegenteilige Fall ist zutreffend: Die zunehmende Durchsetzung wirtschaftlicher Zielsetzungen und Kalküle im patientenbezogenen Denken und Handeln steht im Widerspruch zur beruflichen Motivation, aus der heraus viele Pflegende ihren Beruf praktizieren. Die fortschreitende Ökonomisierung der ursprünglich ethisch-moralischen Grundlagen des Pflegeberufs führen bei vielen Pflegekräften zu persönlichen und teilweise auch fachlichen Dilemmata und bewirken eine Infragestellung des Sinns ihrer beruflichen Tätigkeit. Ohne Sinngehalt kann aber gerade die Pflegearbeit als eine „erdrückende Last“ empfunden werden (Käppeli 2006).

\section{Ausblick}

Die Ökonomisierung des Gesundheitswesens geht weiter - und die Krankenhauslandschaft in Deutschland wird auch künftig in Bewegung sein: Auf der einen Seite wird die Privatisierung der Krankenhäuser, vor allem die Etablierung von privaten Klinikketten, vorangehen. Auf der anderen Seite wird sich das „Kliniksterben“ fortsetzen, und zahlreiche Kommunen werden die Pforten ihrer Krankenhäuser endgültig schließen. Ein kritischer Meilenstein für viele Kliniken wird der Abschluss der DRGKonvergenzphase Ende 2008 sein. Der finanzielle Druck wird mit der geplanten landesweiten Gleichschaltung der Fallpauschalen (also der vollständigen Aufhebung noch bestehender klinikindividueller Fallpauschalen) zunehmen, mit der Konsequenz eines weiteren Abbaus von Betten und Planstellen. Dabei haben die Kliniken in Deutschland bereits in den vergangenen Jahren erhebliche Leistungssteigerungen erzielt: Während der letzten zehn Jahre ist die Zahl der Betten um $13 \%$ gesunken, die Aufenthaltsdauer der Patienten wurde um $24 \%$ reduziert und die Anzahl der stationär behandelten Fälle um $12 \%$ erhöht (Laschet 2007).

Für die Beschäftigten in Krankenhäusern sind diese Zahlen zugleich Ausweis ihrer veränderten Arbeitssituation: Falzahl, Verweildauer und Bettenanzahl sind nicht nur relevante Kennziffern für die Ermittlung der Effizienz von Krankenhäusern, sondern zugleich zentrale Stellschrauben für die Leistungsverausgabung der Beschäftigten. Nicht nur die betroffenen Pflegekräfte, sondern auch ihre Vorgesetzten berichten von einer negativen Veränderung der Arbeitssituation im pflegerischen Dienst und sorgen sich, ob die existierenden Arbeits- und Leistungsbedingungen von Beschäftigten überhaupt bis zum Renteneintrittsalter bewältigt werden können. Die Arbeitsbelastungen der Pflegekräfte sind an die Grenze des $\mathrm{Zu}$ mutbaren gestiegen. Im Zuge der fortschreitenden Ökonomisierung wandelt sich aber nicht nur ihre Arbeitssituation, auch ihre berufliche Identität wird in ihrem innersten Kern davon berührt. Die hohen ethisch-moralischen Ansprüche der Pflegekräfte an ihre Arbeit geraten in einen systematischen Konflikt mit den aktuellen Ökonomisierungstendenzen der Krankenhausarbeit. Im Mittelpunkt ihrer beruflichen Aufmerksamkeit steht eben nicht die Gewinnoptimierung, sondern der Patient, und in manchen Fällen auch dessen individuelles Schicksal. Für eine in ihren Augen "gute Pflege" fehlt ihnen aber aufgrund der steigenden Arbeitsverdichtung und Bürokratisierung ihrer Arbeit immer häufiger schlichtweg die notwendige Zeit. Das wirkt sich auch auf die Qualität der pflegerischen Beziehungen zwischen Pflegenden und Pflegebedürftigen aus, die unter dem zunehmenden ökonomischen Druck zu Kurzkontakten verkümmern können.

Gegenwärtig lässt sich noch nicht prognostizieren, welche Folgen diese „Widerspruchskonstellation“ (Boes/Baukrowitz 2002) für die Pflegekräfte und ihr Interessenhandeln haben werden. Die Befunde verdeutlichen allerdings, dass die Sicherung und Durchsetzung von Kriterien „guter Pflege“ ein zentrales Gestaltungsfeld der Zukunft darstellt, das trotz der wach- 
senden Einkommens- und Beschäftigungsunsicherheiten im Pflegebereich nachhaltig in den Blick zu nehmen ist. Die Relevanz dieses arbeitspolitischen Handlungsfelds ergibt sich auch aus der Spezifik dieser personenbezogenen Dienstleistungsarbeit. Im Unterschied zur Arbeit in anderen Wirt- schaftssektoren ist der Hauptarbeitsgegenstand von Pflegekräften der Mensch. Keine andere Berufsgruppe im Krankenhaus steht in so intensivem Kontakt mit den Patienten wie die Pflegkräfte. Es wäre also ein Irrtum, davon auszugehen, dass ihre Arbeitssituation auf Dauer ohne Einfluss auf die Versorgungsqualität der Patienten bleibt - im positiven wie im negativen Sinne. Über kurz oder lang werden die Wirkungen des gegenwärtigen Strukturwandels, wenn dem nicht entgegengesteuert wird, nicht nur für die direkt davon betroffenen Pflegekräfte spürbar sein.

\section{LITERATUR}

Badura, B. (1990): Interaktionsstress. Zum Problem der Gefühlsregulierung in der modernen Gesellschaft, in: Zeitschrift für Soziologie 5, S. 317328

Bartolomeyczik, S. (2007): Reparaturbetrieb Krankenhaus. DRGs und ihre Auswirkungen aus Sicht der Pflege, in: Dr. med. Mabuse 166, S. 57-60 Boes, A. (2005): Informatisierung, in: SOFI/ IAB/ISF München/INIFES (Hrsg.): Berichterstattung zur sozioökonomischen Entwicklung in Deutschland - Arbeits- und Lebensweisen. Erster Bericht, Wiesbaden, S. 211-244

Boes, A./Baukrowitz, A. (2002): Arbeitsbeziehungen in der IT-Industrie. Erosion oder Innovation der Mitbestimmung, Berlin Braun von Reinersdorff, A. (2002): Strategische Krankenhausführung. Vom Lean Management zum Balanced Hospital Management, Bern u.a. Braun, B./Müller, R. (2006): Versorgungsqualität im Krankenhaus aus der Perspektive der Patienten. Ergebnisse einer wiederholten Patientenbefragung und einer Längsschnittanalyse von GEK-Routinedaten, GEK Edition, Schriftenreihe zur Gesundheitsanalyse, Band 46, St. Augustin

Braun, B./Müller, R./Timm, A. (2004): Gesundheitliche Belastungen, Arbeitsbedingungen und Erwerbsbiographien von Pflegekräften im Krankenhaus, GEK Edition, Schriftenreihe zur Gesundheitsanalyse, Band 32, St. Augustin

Büssing, A. (1997): Neue Entwicklungen in der Krankenpflege. Reorganisation von der funktionalen zur ganzheitlichen Pflege, in: Büssing, A. (Hrsg.): Von der funktionalen zur ganzheitlichen Pflege. Reorganisation von Dienstleistungsprozessen im Krankenhaus, Göttingen, S. 15-48 Dörre, K./Röttger, B. (Hrsg.) (2003): Das neue Marktregime - Konturen eines nachfordistischen Produktionsmodells, Hamburg Dunkel, W. (2005): Zur Lebensführung von Pflegekräften, in: Schroeter, K. R./Rosenthal, T. (Hrsg.): Soziologie der Pflege. Grundlagen, Wissensbestände und Perspektiven, Weinheim, München, S. 227-246 Hartwig, R. (2002): Vergütung im Krankenhaus. Leistungsorientierte Vergütung statt BAT - Gesichtspunkte einer Reform, in: Arnold, M./Klauber, J./Schellschmidt, H. (Hrsg.): Krankenhausreport 2001, Schwerpunkt: Personal, Stuttgart/New York, S. 133-140

Käppeli, S. (2006): Das therapeutische Bündnis der Medizin und Pflege wie lange noch?, in: Schweizerische Ärztezeitung 26, S. 1221-1225
Kühn, H. (2003): Ethische Probleme der Ökonomisierung von Krankenhausarbeit, in: Büssing, A./Glaser, J. (Hrsg.): Dienstleistungsqualität und Qualität des Arbeitslebens im Krankenhaus, Schriftenreihe Organisation und Medizin, Göttingen u. a., S. 77-98

Kühn, H./Klinke, H. (2006): Krankenhaus im Wandel. Zeit- und Kostendruck beeinflussen die Kultur des Heilens, in: WZB-Mitteilungen 113, S. 6-9

Kühn, H./Simon, M. (2001): Anpassungsprozesse der Krankenhäuser an die prospektive Finanzierung (Budgets, Fallpauschalen) und ihre Auswirkungen auf die Patientenorientierung, Abschlussbericht, Berlin Laschet, H. (2007): Medizin im Krankenhaus muss schlanker und schneller werden - sonst ist der Ruin programmiert. Ärzte Zeitung, 25.01. (http://www.aerztezeitung.de/docs/2007/01/25/014a0203.asp?cat=/ politik/gesundheitssystem uns)

Michaelis, M. (2005): Pflege als extreme Verausgabung: arbeitssoziologische Aspekte, in: Schroeter, K. R./Rosenthal, T. (Hrsg.): Soziologie der Pflege. Grundlagen, Wissensbestände und Perspektiven, Weinheim/ München, S. 263-277

Pfaff, H./Lütticke, J./Badura, B./Piekarski, C./Richter, P. (Hrsg.) (2004): "Weiche" Kennzahlen für das strategische Krankenhausmanagement. Stakeholderinteressen zielgerichtet erkennen und einbeziehen, Bern u.a. Rieder, K. (1999): Zwischen Lohnarbeit und Liebesdienst. Belastungen in der Krankenpflege, Weinheim/München

Sauer, D. (2005): Arbeit im Übergang. Zeitdiagnosen, Hamburg

Tewes, R. (2006): Primary Nursing. "Das Pflegesystem der Zukunft", in: Die Schwester Der Pfleger 2, S. 88-90

Tondorf, K. (2007): Tarifliche Leistungsentgelte - Chance oder Bürde?, Berlin

Vogd, W. (2006): Die Organisation Krankenhaus im Wandel. Eine dokumentarische Evaluation aus Sicht der ärztlichen Akteure, Bern u.a.

Wagner, H. (Hrsg.) (2005): "Rentier' ich mich noch?“" Neue Steuerungskonzepte im Betrieb, Hamburg

Zimber, A. (2003): Kranke Pflege. Belastungen im Pflegeberuf und ein möglicher Ansatz zur Gesundheitsförderung, in: Dr. med. Mabuse 142, S. $38-40$ 